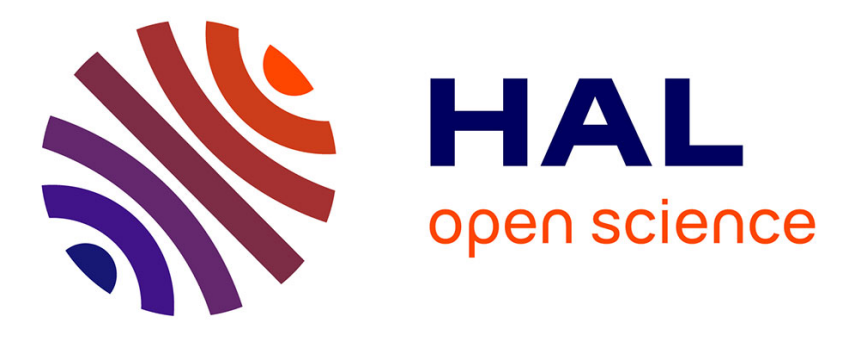

\title{
New methodology to define roller geometry on power bearings
}

Emmanuel Mermoz, Douchane Fages, Laurent Zamponi, Jean-Marc Linares, Jean-Michel Sprauel

\section{To cite this version:}

Emmanuel Mermoz, Douchane Fages, Laurent Zamponi, Jean-Marc Linares, Jean-Michel Sprauel. New methodology to define roller geometry on power bearings. CIRP Annals - Manufacturing Technology, 2016, 65 (1), pp.157-160. 10.1016/j.cirp.2016.04.095 . hal-01454812

\section{HAL Id: hal-01454812 https://hal.science/hal-01454812}

Submitted on 8 May 2020

HAL is a multi-disciplinary open access archive for the deposit and dissemination of scientific research documents, whether they are published or not. The documents may come from teaching and research institutions in France or abroad, or from public or private research centers.
L'archive ouverte pluridisciplinaire $\mathbf{H A L}$, est destinée au dépôt et à la diffusion de documents scientifiques de niveau recherche, publiés ou non, émanant des établissements d'enseignement et de recherche français ou étrangers, des laboratoires publics ou privés. 


\title{
New methodology to define roller geometry on power bearings
}

\author{
Emmanuel Mermoz (3) ${ }^{\mathrm{a}, \mathrm{b}} *$, Douchane Fages ${ }^{\mathrm{a}}$, Laurent Zamponi ${ }^{\mathrm{a}}$, Jean-Marc Linares (1) ${ }^{\mathrm{b}}$, Jean-Michel \\ Sprauel b
}

${ }^{a}$ AIRBUS HELICOPTERS, Aéroport de Marseille Provence, 13700 Marignane, France ${ }^{b}$ Aix-Marseille Université, CNRS, ISM UMR 7287, 13288 Marseille, Cedex 09, France.

This paper presents new tools developed to improve the geometrical corrections required on roller bearings of Helicopter gearboxes suffering from heavy deflection. A complex non-linear finite element model of the whole gearbox is firstly implemented to identify the deflection of inner and outer raceways under operational loads. The computed misalignment is then used as an input to predict and check the contact pressure edge effects occurring in the contact area and iteratively optimize the roller shape. Several approaches are tested based either on Boussinesq's potential theory or specific FEM calculations. They permit increasing the service life of the bearings while reducing the overall gearbox development lead-time.

Keywords: Optimization, Finite Element Method, Bearings

\section{Introduction}

Helicopter gearboxes are suffering from severe deflections in operation that are resulting from the heavy loads needed to drive the main rotor in combination with the necessity to minimize weight on all parts. This overall deflection generates relative angular displacement between inner and outer races of power bearings. The consequence on roller bearings is mostly a magnification of the contact pressure at the extremity of the roller contact area as illustrated by Figure 1 .

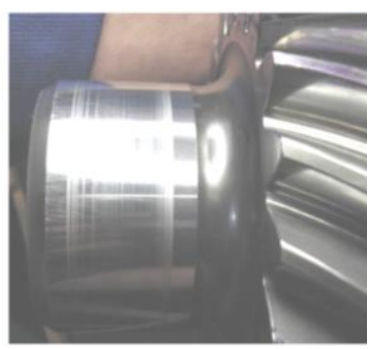

Figure 1. Typical example of overpressure marks on roller bearing integrated raceway.

This phenomenon is often called "edge effects" and can lead, pending on loads or numbers of cycles, to a more severe bearing degradation, a spalling. Spalling induces generation of particles that are detected by chip detectors until finally leading to a removal from service of the gearbox. Mastering the apparition of "edge effects" on power bearing is thus mandatory to guaranty the reliability of helicopters gearbox in operation.

Quite often the contact between the rolling elements and the raceway is modelled by Hertz theory.

This modelling is very effective to know the load distribution in the bearing, but is not sufficient to know the pressure distribution on the contact line. In fact, Hertz theory, assuming for contact of an infinite cylinder on a plane, is a twodimensional application that does not account for boundary conditions.

Indeed, at the ends of the cylinder, it appears a localized deflection's gradient at the surface that induces tensions in the material which increases the contact pressure. Figure 2 gives some representations of the pressure distribution along the contact line considering edge effect (a) or not (b). 


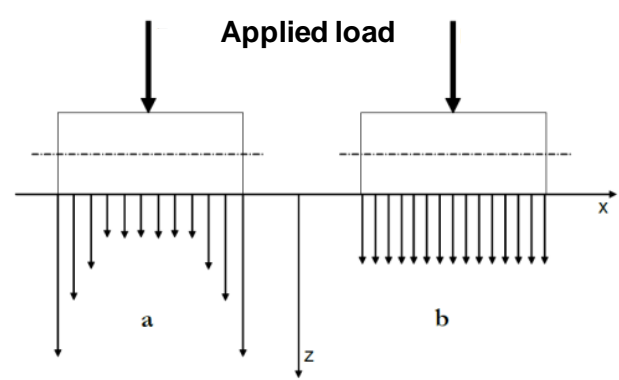

Figure 2. Illustration of the edge effect on the pressure distribution.

To avoid excessive pressure at the edges, bearing manufacturers apply geometrical correction on the rollers. So these are no more completely cylindrical over their entire length. The edges of the rollers are undercut. The shape used the more in helicopter power roller bearing is illustrated in Figure 3, with the introduction of a circular modification of the profile. However, the values of the relief cut radius (R) and connecting radius (r) are often defined from the past experience of bearing manufacturers. This value depends on the length of the roller and on the type of application. In helicopter gearboxes suffering from heavy deflection, these empirical rules are not sufficient to avoid edges effects as shown by Figure 2.

\section{Several methods to simulate contact pressure distribution}

When the contact between mechanical parts is localized, Hertz theory is still widely used [1, 2]. It is based on the evaluation of the equivalent curvature of parts and the contact surface. The pressure and stress fields are then computed.

Various methods exist as an alternative to Hertz theory, such as the finite element methods [3, 4, 5]. However, the localized character of the problem requires extremely fine volumes of meshing and delicate treatment of the contact conditions. These constraints can lead to simulations requiring high calculation times if this process is not handled carefully. To overcome this situation, two semi analytical approaches have been first studied by the authors. The first one is based on semi analytic Punch calculations, whereas the other one is based on Boussinesq's theory of potential.

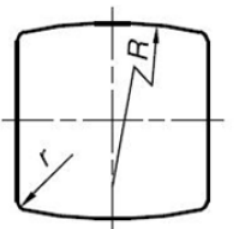

Figure 3. Illustration of roller geometry correction to avoid edge effect.

\subsection{Analytical simulation of the pressure distribution based on "punch" modeling}

For some specific geometrical shapes, it is possible to find analytical solutions to contact problems. In the publication of Johnson [6] or Hill [7] several types of "punch" are studied. All treated cases are reduced to a study in two dimensions using the assumptions of symmetry, plane strain and stress. The longitudinal profile of the rollers used in helicopter transmissions boxes (Figure 3) is close to the punch model studied by Goryacheva [8], except at the junction, where there is no tangency between the straight portion and the circular one. The equations proposed by Goryacheva have thus been modified to comply with the profile of the new punch as defined in Figure 4 . We determine in this way the pressure distribution along the median slice. This function is then extended over the entire contact width (x-axis) using Hertz's theory.

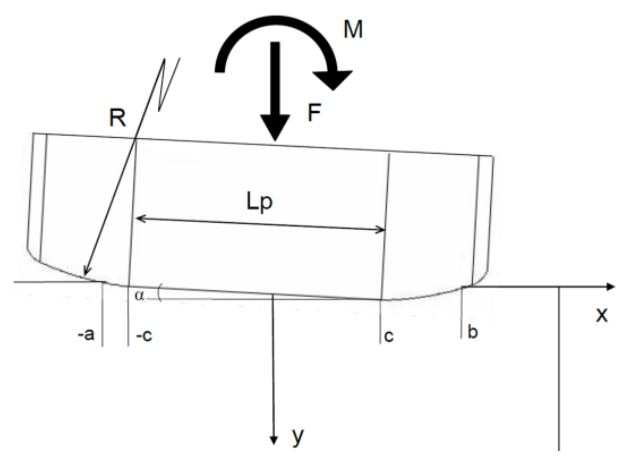

Figure 4. Illustration of the punch models parameters. 
The 3D contact problem is reduced first to a 2D problem in the median slice, which allows calculating the linear distribution of forces and moments along the contact line. Then the pressure field is estimated by the punch method. Following this resolution, we get the length of contact, the tilt angle of the punch and the pressure distribution.

From the previous results on the middle slice, the contact width in the perpendicular direction can be determined. In the case of a contact between two rollers, Hertz model gives a 2D parabolic distribution, which is used to set a contact width according to the maximum pressure in the computed section. It allows modeling the pressure field in three dimensions. However, the distribution of forces and moments applied has to be estimated from the new pressure field. For this, two mathematical expressions have to be calculated: the integral of the pressure on the contact surface and the integral of the product of the pressure and the distance to the center of the contact surface. These two integrals are calculated numerically. As long as the convergence limits are not reached, the program iterates by changing the values of forces and moments along the length of the roller. The results obtained are the contact length, the contact width, the angle of inclination and the 3D pressure field as described by Figure 5.

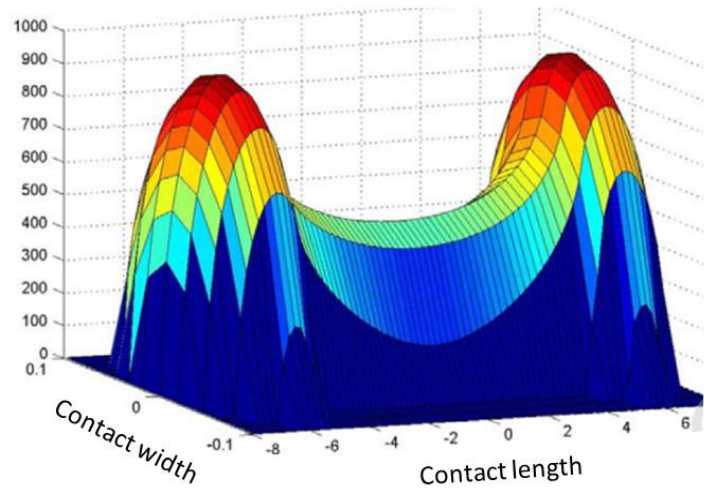

Figure 5. Illustration of 3D pressure distribution of a roller bearing based on Punch and Hertz modeling (computed for $\alpha=0^{\circ}$ ) [9].

\subsection{Usage of Boussinesq's theory of potential and Fast Fourier Transform (FFT)}

The method presented in previous paragraph is based on several hypotheses like symmetry or plane strain states. In order to overcome these limitations, the Boussinesq and Cerruti theory of potentials [10,11] has been considered as a basic framework for contact pressure estimation. This approach was used by Goryacheva [8] in the case of a rounded rigid punch, invariant in one direction, and indented with partial slip. He resolved the problem using integral equations and highlighted the existence of excess pressures close to the changes in curvature of the punch. This theory has been treated here in three dimensions. As a first approach, only the case of a purely normal surface contact load has been considered. The punch was also assumed perfectly rigid.

In our approach, the mathematical resolution is based on a Fourier deconvolution method. Such approach has already been discussed by several other teams $[12,13]$. However they only dealt with the case of a contact between a ball and a half space and proposed solutions which are limited by the domains of computing. The resolution of the contact problem by using the Fourier space has therefore been improved. In our method, the contact pressure, displacement field, strain and stress distributions induced by localized elastic contacts between parts are computed using Boussinesq's theory of potentials.

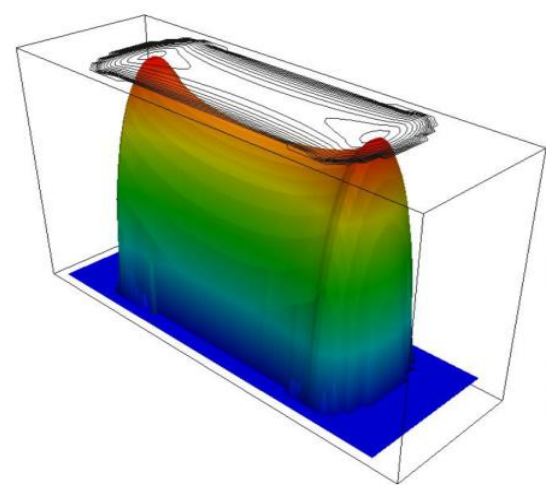

Figure 6. 3D view of the pressure fields generated by the contact of the roller on a raceway based on Boussineq's theory. 
For that purpose it has been demonstrated that each of these elastic fields can be written as convolution products of the surface pressure distribution, thus permitting using Fast Fourier analysis to solve the contact problem. However, on the contrary to classical approaches, where the shape of the contact pressure is assumed to be well known, here, the only inputs are the geometry, elastic constants and penetration of the punch. The boundary of the contact surface is thus completely undefined.

An iterative algorithm, based on an active contour model, as employed in image processing, has therefore been developed to define the contact area. This permitted computing the displacement, strain and stress at any point inside the elastic body. The method has first been applied to simple contacts. The results were then compared to classical Hertz solution. The algorithm was also applied to a contact similar to a roller on a raceway, as illustrated by Figure 6.

In that case, the results were compared to Finite Elements calculations. In order to avoid just comparing different models, an experimental protocol has also been set up as described in [14]. Chromatic confocal optical measurements were thus carried out successfully to characterize the surface displacement field of transparent discs of polymethyl methacrylate, indented by an aspherical punch. The method developed allowed evaluating the distribution of pressures applied to the contact area and the strains and stresses at any point within the material. The experimental results were in very good agreement with the predicted results.

Nevertheless, during the time used to investigate semi analytical formulations to estimate contact pressure, the computer performance available in industry has strongly increased. As the nonlinear solvers were also improving FEM prediction capacities, analytical techniques investigated by the authors have been combined to FEM approaches to implement a new hybrid methodology at Airbus Helicopters.

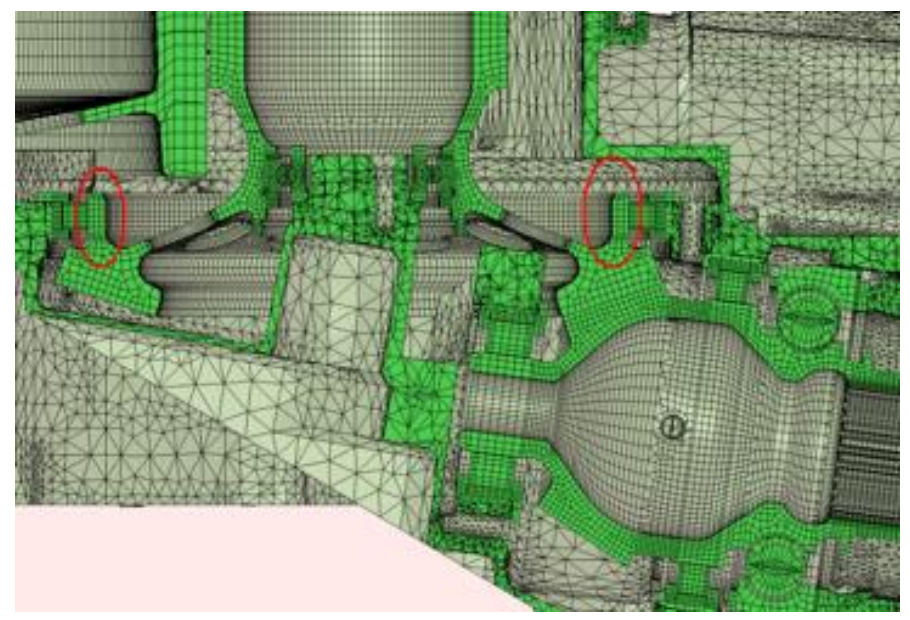

Figure 7. Partial view of the FEM model used to estimate the load distribution and geometrical deflection of the entire parts.

\section{A four-step hybrid methodology used in industrial context}

\subsection{Estimation of relative deflection between inner and outer race}

As one of the most important contributing factors of the creation of edge effect on roller bearings is the misalignment between inner and outer races, the first step consists in accurately computing the deflection of the different components of the gearbox which is therefore considered as a global system.

For that purpose, specific nonlinear FEM models have been developed as illustrated by Figure 7. This first step obviously included the entire parts of the gearbox: casing, shafts, gears, bearings. It demonstrated that the loads applied to the bearings and the total deflection between inner and outer race is greatly impacted by the stiffness of all the components.

\subsection{Estimation of the loaded area and of the local deflection of bearing raceways}

Secondly, the FEM elements used to describe the contacts inside the different components of the bearings allow capturing the loads transferred to each roller. A polar diagram can thus be computed to identify the loaded area and to characterize the force applied to each roller (Figure 8). A complete meshing of the inner and outer races allows thus estimating the relative radial and angular deflections at each azimuthal position. 

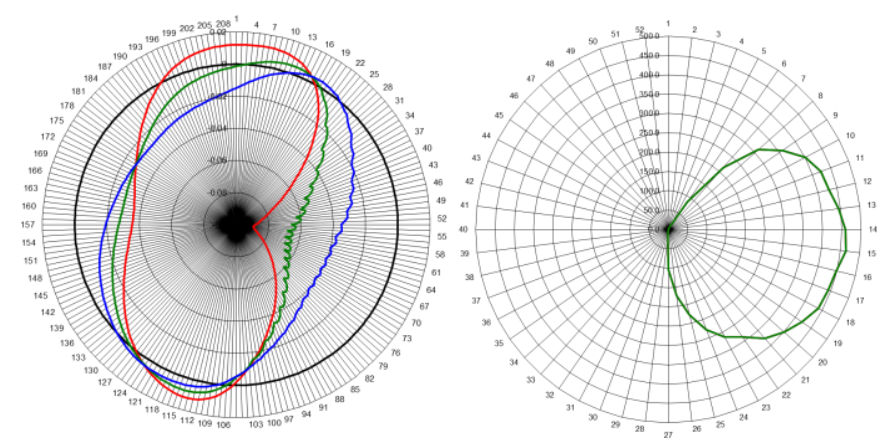

Figure 8. Polar diagrams representing the load distribution on each roller (left) and the inner and outer raceway deflections (right).

\subsection{Pressure distribution on the most loaded areas}

Thirdly, after having localized the most critical azimuthal position, the load of each roller and the relative position of inner or outer races are used as inputs to feed another local FEM model. A specific parametric meshing has therefore been developed to reach a balance between accuracy and computing time (Figure 9).

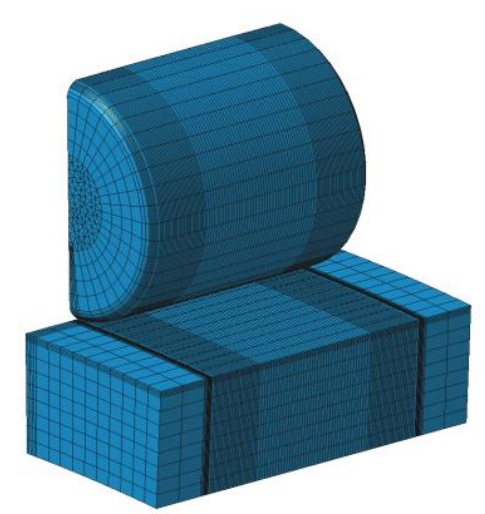

Figure 9. Illustration of the parametric meshing used for the estimation of the contact pressure distribution by nonlinear FEM calculations.

As represented by Figure 10, the local FEM results are then treated automatically to obtain the distribution of the contact pressure on the most loaded section of the roller. Four curves are presented corresponding to different conditions of loading. "Ref load" and "Max load" curves illustrate an increase of torque of about 40\% that amplifies the edge contact pressure by around $90 \%$. Without accounting for the nonlinear misalignment of the raceways, the pressure increase would however be estimated to $18 \%$.

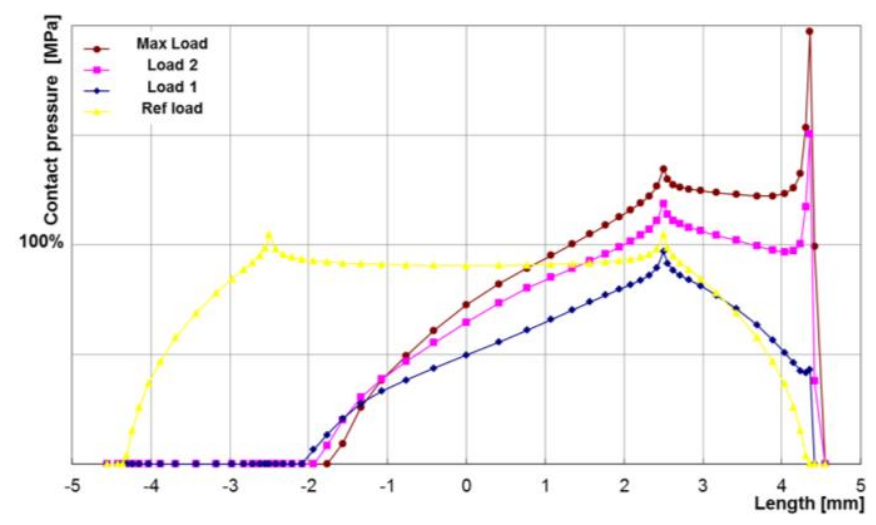

Figure 10. Contact pressure distribution along the roller edge.

Such high pressure values have been confirmed by bench tests performed on a helicopter gearbox during development phase. A spalling of the outer race occurred, starting at the end of the contact zone of the roller (Figure 11). 


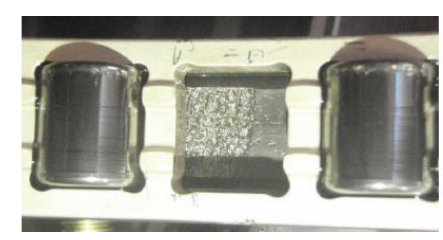

Figure 11. Example of outer spalling detected in a bearing tested with heavy load and significant outer and inner raceway misalignment.

\subsection{Correcting the micro geometry of the roller}

The hybrid methodology allows finally modifying the geometry of the rollers, until reaching a shape that complies with the criteria of dimensioning corresponding to the most critical loading conditions applied to the bearings. To increase the capability of the roller to bear large misalignments, the value of the relief cut radius (R) had thus to be modified. It resulted in a reduction of the linear length of the roller. In normal conditions of loading, this would obviously increase the contact pressure of about $10 \%$. In case of heavy misalignment, on the contrary, it allowed a complete removal of the overpressure peaks (Figure 12).

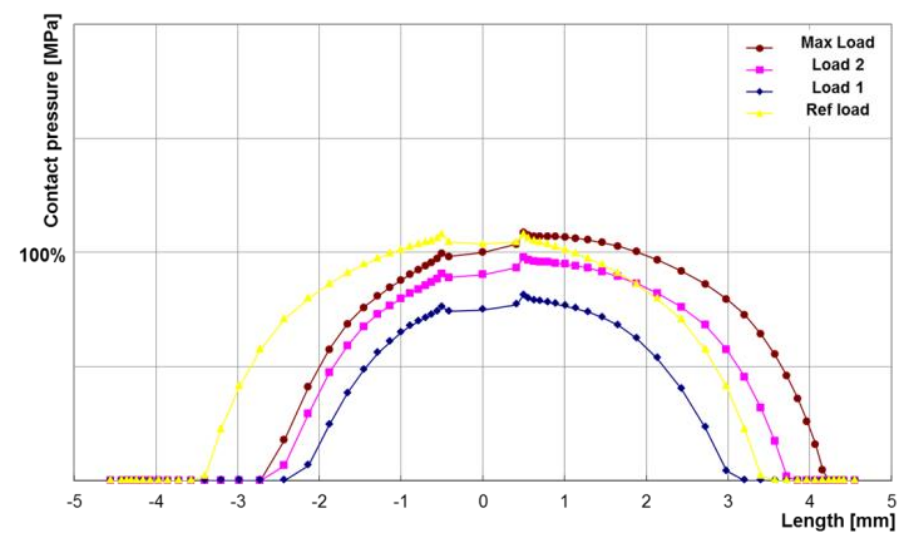

Figure 12. Contact pressure distribution along the edge of a roller after correction of the micro geometry.

New bearings were designed containing rollers with the corrected micro geometry. Due to the heavy loads carried by the gearboxes, integrated raceways were necessary to avoid fretting corrosion. These surfaces were therefore machined and ground directly by Airbus Helicopters workshop. The new improved rollers and bearing cages were realized by the bearing supplier of the company. A special manufacturing process was developed for that purpose. After turning and grinding, different crown honing operations were applied to the rollers. The rollers are finally polished by trumbling. The bearings were tested in the same loading conditions than for the experiments of Figure 11. It demonstrated the absence of any edge effect existing in overload tests (Figure 13).

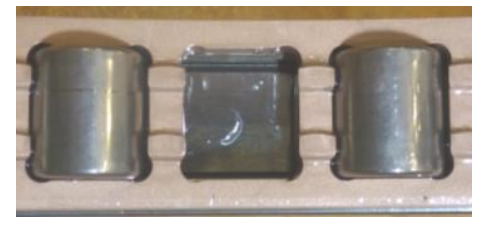

Figure 13. Behaviour of bearings redesigned with the proposed methodology and analyzed after endurance tests performed in the same loading conditions than for figure 11.

\section{Conclusion}

Several methods to improve the prediction of the contact pressure distribution on power bearings have been investigated by the authors. They were either based on punch approaches, Boussinesq's theory of potentials computed through FFT processing or nonlinear FEM analysis. The result of that work is the implementation in an industrial context of a new hybrid methodology that has successfully demonstrated its efficiency by comparison to several kinds of bench tests. This four step methodology is firstly based on the analysis of a complex non-linear FEM model that allows estimating the loads on each roller and the relative misalignment between inner and outer races. The outputs from this simulation are then used to perform a local nonlinear FEM computation with heavy meshing density in order to estimate the contact 
pressure distribution on the most critical roller. This local FEM modelling allows quick iterations on the micro geometry of the roller to reach the best design.

\section{References}

[1] Gonzalez-Perez, I., Iserte, J.L., Fuentes, A., 2011, Implementation of Hertz theory and validation of a finite element model for stress analysis of gear drives with localized bearing contact, Mechanism and Machine Theory, 46/6: 765-783.

[2] Zhupanska, O.I., 2011, Contact problem for elastic spheres: Applicability of the Hertz theory to non-small contact areas, International Journal of Engineering Science, 49/7: 576-588.

[3] Kania, L., 2006, Modelling of rollers in calculation of slewing bearing with the use of finite elements, Mechanism and Machine Theory, 41/11:13591376.

[4] Laniado-Jácome, E., Meneses-Alonso, J., Diaz-López V., 2010, A study of sliding between rollers and races in a roller bearing with a numerical model for mechanical event simulations, Tribology International, 43/11: 2175-2182.

[5] Wei, Y., Qin, Y., Balendra, R., Jiang, Q., 2004, FE analysis of a novel roller form: a deep end-cavity roller for roller-type bearings, Journal of Materials Processing Technology, 145/2: 233-241.

[6] Johnson, K.L., 1985, Contact Mechanic, Cambridge University Press.

[7] Hills, D.A., Nowell, D., Sackfield, A., 1993, Mechanics of Elastic Contacts, Butterworth-Heinemann ltd, Oxford.

[8] Goryacheva, I.,G., Murthy, H., Farris, T.N., 2002, Contact problem with partial slip for the inclined punch with rounded edges, International Journal of fatigue, 24: 1191-1201.

[9] Zamponi, L., Mermoz, E., Linares, J.M., Sprauel, J.M., 2009, Impact of geometrical defects on bearing assembly with integrated raceways on the aeronautical reducers, International Journal of Mechanism and Machine Theory, 44:1108-1120.

[10] Boussinesq, J., 1885, Application des Potentiels à l'étude de l'équilibre et du mouvement des solides élastiques, Paris: Gauthier-Villars.

[11] Cerruti, V., 1882, Mem. fis. mat, Roma, Acc Lincei.

[12] Liu, S., Hua, D., Chen, W.W., Wang, Q. J., 2007, Tribological modeling: Application of fast Fourier transform, Tribology International, 40:1284-1293.

[13] Andersson, J., Almqvist, A., Larsson, R., 2011, Numerical simulation of a wear experiment, Wear, 271/11-12: 2947-2952.

[14] Solle, J., Linares, J. M., Sprauel, J. M., Mermoz, E., 2012, Optical measurement for the estimation of contact pressure and stress , CIRP Annals, 61/1:483-486. 